\title{
RESISTÊNCIA NATURAL DE NOVE MADEIRAS DO SEMI-ÁRIDO BRASILEIRO A FUNGOS CAUSADORES DA PODRIDÃO-MOLE ${ }^{1}$
}

\author{
Juarez Benigno Paes², Verlândia de Medeiros Morais ${ }^{3}$ e Carlos Roberto de Lima²
}

\begin{abstract}
RESUMO - O objetivo desta pesquisa foi avaliar a resistência de nove madeiras de ocorrência no semi-árido brasileiro a fungos de podridão-mole, em condições de laboratório. As madeiras estudadas foram algaroba (Prosopis juliflora), angico (Anadenanthera colubrina var. cebil), aroeira (Myracrodruon urundeuva), braúna (Schinopsis brasiliensis), cássia (Senna siamea), craibeira (Tabebuia aurea), cumaru (Amburana cearensis), pau-d'arco (Tabebuia impetiginosa) e pereiro (Aspidosperma pyrifolium). De cada espécie foram retirados corpos-deprova de 3,0 x 1,5 x 0,5 cm, com a maior dimensão no sentido das fibras, em quatro posições na direção medula-casca do tronco. As amostras permaneceram por 120 dias sob a ação da microflora natural existente em solo orgânico. A resistência ao apodrecimento da aroeira, braúna e cássia não foi afetada pela posição na direção medula-casca, não esteve relacionada à densidade das madeiras ensaiadas. As madeiras de aroeira e braúna (cerne), pereiro e pau-d' arco apresentaram melhor desempenho. A resistência natural não esteve associada à concentração de extrativos solúveis em água quente.
\end{abstract}

Palavras-chave: Madeiras do semi-árido, resistência natural e podridão-mole.

\section{NATURAL RESISTANCE OF NINE WOODS OF BRAZILIAN SEMI-ARID REGION TO SOFT-ROT FUNGI}

\begin{abstract}
The objective of this research was to evaluate the wood natural resistance of nine species of woods of the Brazilian semi-arid region to soft-rot fungi, under laboratory conditions. The studied woods were Prosopis juliflora, Anadenanthera colubrina var. cebil, Myracrodruon urundeuva, Schinopsis brasiliensis, Senna siamea, Tabebuia aurea, Amburana cearensis, Tabebuia impetiginosa and Aspidosperma pyrifolium. Specimens measuring $3.0 \times 1.5 \times 0.5 \mathrm{~cm}$, with the largest dimension taken in the fiber direction, were obtained from four positions from pith to bark in the trunk. The specimens were submitted to action of organic soil natural micro-flora for 120 days. The wood resistance to soft-rot of Myracrodruon urundeuva, Schinopsis brasiliensis and Senna siamea was not affected by the pith to bark position, and was not associated to wood density. The Myracrodruon urundeuva and Schinopsis brasiliensis heartwood, and Aspidosperma pyrifolium and Tabebuia impetiginosa woods were the most resistant to the fungi. The wood natural resistance was not associated to the content of hot water soluble extractives.
\end{abstract}

Key words: Brazilian semi-arid woods, wood natural resistance and soft-rot fungi.

\footnotetext{
${ }^{1}$ Recebido em 03.10.2003 e aceito para publicação em 20.04.2005.

${ }^{2}$ Departamento de Engenharia Florestal/CSTR/UFCG - Campus de Patos - Cx. Postal 64 - 58700-970 PatosPB.E-mail:<jbp2@uol.com.br > e <crlima16@bol.com.br >, respectivamente.

${ }^{3}$ Acadêmica de Engenharia Florestal e bolsista PIBIC/UFPB/CNPq-Cx. Postal 64-58700-970Patos-PB.E-mail:<verlandiam@bol.com.br.>.
} 


\section{INTRODUÇÃO}

O semi-árido brasileiro abrange uma área de 1.150.662 $\mathrm{km}^{2}$, que corresponde a 74,30\% da Região Nordeste e a 13,52\% da superfície do Brasil (PNUD/FAO/IBAMA/ GOV. PARAÍBA, 1994). A vegetação do semi-árido (Caatinga) é composta de uma variedade de espécies arbustivas e arbóreas que apresentam potencial pouco conhecido cientificamente.

A madeira oferece uma gama variável de utilização nos meios rural e urbano. Porém, em virtude da sua estrutura e constituição química, sofre o ataque de vários organismos capazes de causar sua deterioração. Dentre esses organismos, os fungos são os responsáveis pelos maiores danos (HUNT e GARRATT, 1967, CAVALCANTE, 1982; CARBALLEIRA LOPEZ e MILANO, 1986).

A constituição química da madeira é bastante variável entre espécies e até mesmo entre partes de uma mesma planta, o que determina sua resistência ao ataque de organismos biológicos, sendo o cerne mais resistente que o alburno (CARBALLEIRA LOPEZ e MILANO, 1986).

Os fungos xilófagos são os organismos responsáveis pelas maiores perdas causadas a estruturas de madeira, como postes, dormentes, moirões e outras. Para avaliar a resistência natural da madeira a fungos, são necessários testes acelerados em laboratório, nos quais amostras de madeira são expostas aos fungos xilófagos causadores das podridões-branca ou parda.

Com relação aos fungos causadores da podridãomole que apresentam características distintas daqueles da podridão-branca ou parda, não se pode aplicar o teste de apodrecimento acelerado (SAVORY, 1972; SAVORY e CAREY, 1973; JANKOWSKY, 1986).

Para esse tipo de ensaio, não há ainda nenhuma metodologia normatizada, porém, como em condições naturais a podridão-mole resulta da ação de diferentes organismos, muitos testes envolvem o soterramento de blocos de madeira em solo ao natural (THEDEN, 1961; THORNTON et al., 1977; HEDLEY et al., 1979; GERSONDE e KERNER, 1984), em vermiculita ou em areia estéril (McKAIG, 1984). Quando se utiliza solo, os microrganismos naturalmente presentes podem ser utilizados na degradação da madeira. Porém, usandose vermiculita ou areia, há a necessidade da inoculação desse substrato com espécies de fungos de reconhecida capacidade de causar podridão-mole (BEHR, 1973; WILKINSON, 1979; CARBALLEIRALOPEZeMILANO, 1986).

Normalmente, a avaliação do ensaio é efetuada por meio da medição da perda de massa durante a exposição, acompanhada do exame microscópio de alguns corpos-de-prova, para constatação do tipo de apodrecimento (BEHR, 1973; WILKINSON, 1979; JANKOWSKY, 1986).

A resistência ao ataque de organismos xilófagos é afetada pelas condições de uso. Assim, o conhecimento da resistência natural da madeira é de fundamental importância para que se possa recomendar o seu emprego adequadamente, evitar gastos desnecessários com a reposição de peças deterioradas e reduzir os impactos sobre as florestas remanescentes.

Esta pesquisa teve como objetivos avaliar a resistência natural de nove madeiras de ocorrência no semi-árido brasileiro a fungos xilófagos causadores da podridão-mole, em condições de laboratório.

\section{MATERIAL E MÉTODOS}

\subsection{Espécies Estudadas e Confecção dos Corpos-de-Prova}

Nesta pesquisa foram analisadas nove espécies madeireiras, sete de ocorrência natural no semi-árido e duas exóticas (algaroba e cássia) aclimatada na Região Nordeste (Quadro 1).

As espécies testadas, com exceção da algaroba e da cássia, que foram abatidas no Campus da Universidade Federal de Campina Grande (UFCG), em Patos, PB, foram adquiridas em serrarias localizadas nesse mesmo município.

As toras selecionadas foram transformadas em pranchões de $\pm 8 \mathrm{~cm}$ de espessura, que continham $o$ cerne e o alburno intactos. Posteriormente, os pranchões foram desdobrados em vigotas retiradas em quatro posições na direção medula-casca $\left(1^{\mathrm{a}}\right.$. posição interna, $2^{\mathrm{a}}$. posição mediana-interna, $3^{\mathrm{a}}$. posição mediana externa e $4^{\mathrm{a}}$. posição externa), como demonstrado na Figura 1.

Conforme recomendado pelo método IPT/DIMAD D-5 (1980), as vigotas obtidas foram transformadas em corpos-de-prova de 3,0 x 1,5 x 0,5 cm. Posteriormente, foram selecionadas, ao descartar aquelas que apresentavam defeitos, e identificadas em função da espécie, posição na árvore e repetição. 
Quadro 1 - Relação das espécies estudadas

Table 1-List of the studied species

\begin{tabular}{cl}
\hline Nome Comum & Nome Científico \\
\hline Algaroba & Prosopis juliflora D.C. \\
Angico-vermelho & Anadenanthera colubrina (Benth.) Breman. var. cebil (Gris.) Alts. \\
Aroeira & Myracrodruon urundeuva Fr. All. \\
Braúna & Schinopsis brasiliensis Engl. \\
Cássia & Senna siamea L. \\
Craibeira & Tabebuia aurea (Mart.) Bureau. \\
Cumarú & Amburana cearensis (Arr. Cam.) A.C.Smith \\
Pau-d'arco & Tabebuia impetiginosa (Mart.) Standl. \\
Pereiro & Aspidosperma pyrifolium Mart. \\
\hline
\end{tabular}

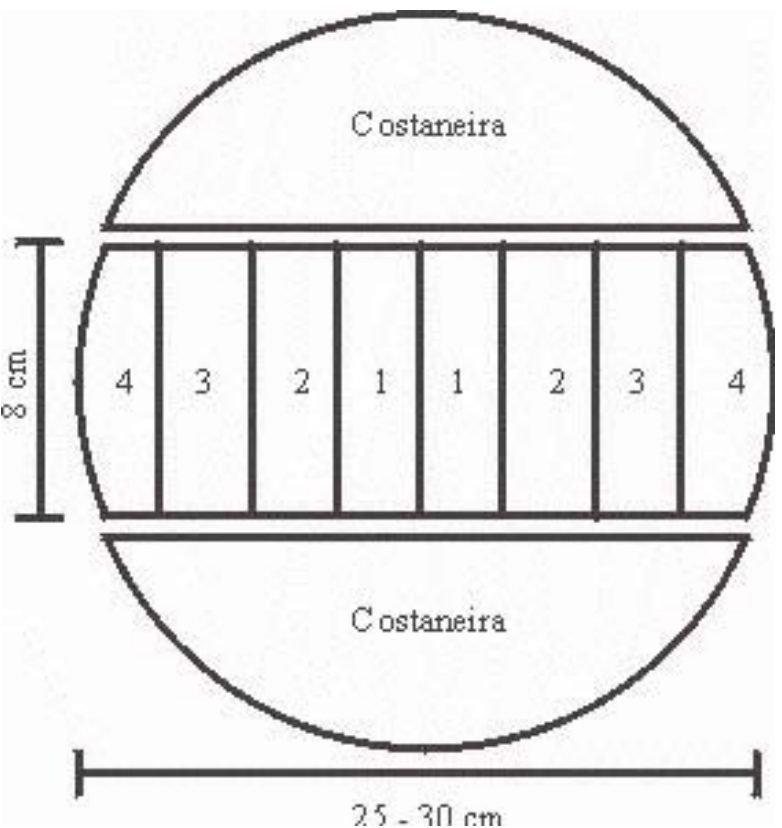

Figura 1 - Obtenção das seções para a confecção dos corposde-prova.

Figure 1 - Preparation of specimens.

Os corpos-de-prova selecionados foram secados em estufa a $103 \pm 2{ }^{\circ} \mathrm{C}$ até massa constante. Mediramse a massa e o volume de cada amostra, conforme o recomendado pela ASTM D - 1413 (1994), bem como os valores utilizados no cálculo da densidade da madeira e da perda de massa causada pelos fungos.

\subsection{Determinação do Teor de Extrativos em Água Quente}

As amostras não selecionadas para o ensaio foram transformadas em cavacos, com dimensões semelhantes a palitos de fósforos, e convertidas em serragem em moinho do tipo Wiley. A serragem obtida foi peneirada, e utilizou-se a que passou pela peneira de 40 meshes e ficou retida na de 60 meshes.

Para a determinação dos extrativos foram seguidas as recomendações da ASTM D-1110 (1994), que padroniza o teste de solubilidade da madeira em água quente. Seguindo as recomendações da norma, foram utilizados 2,0 g de serragem seca ao ar. A serragem foi extraída por três horas à temperatura de ebulição da água. Após a extração, as amostras foram transferidas para cadinhos filtrantes, de porosidade 2 , tarados e submetidas à sucção.

As amostras foram lavadas (cinco vezes) com água destilada quente e secas em estufa à temperatura de $103 \pm 2^{\circ} \mathrm{C}$, por 24 horas, e sua massa, medida em balança de $0,0001 \mathrm{~g}$ de precisão. Todas as extrações foram realizadas em duplicatas.

\subsection{Resistência Natural da Madeira a Fungos de Podridão-Mole}

Para a avaliação da resistência natural das madeiras foram seguidas as recomendações do método IPT/ DIMAD D-5 (1980). A técnica preconiza que as amostras de madeira sejam submetidas à ação da microflora natural existente em solos orgânicos (solos de jardins). Conforme as recomendações, os corpos-de-prova foram soterrados em solo orgânico ( $\mathrm{pH}$ de 6,8 e capacidade de retenção de água de $27 \%$ ) retirado em jardins, no Campus da UFCG em Patos, PB.

Para a montagem do ensaio, utilizaram-se frascos de $500 \mathrm{~mL}$, preenchidos com $350 \mathrm{~g}$ de solo. O solo teve sua umidade ajustada para de $80 \%$ da capacidade de retenção de água pela adição $80 \mathrm{~mL}$ de água destilada. Aos frascos, assim preparados, foram adicionadas quatro amostras de madeira (uma de cada posição no tronco), que permaneceram por 120 dias em sala climatizada (27 $\pm 1{ }^{\circ} \mathrm{Ce} 75 \pm 5 \%$ de umidade relativa). O teste preconiza

R. Árvore, Viçosa-MG, v.29, n.3, p.365-371, 2005 
que sejam utilizadas 10 amostras por tratamento; seis devem ser soterradas em solo ao natural e quatro, em solo esterilizado. Segundo as recomendações, o solo foi esterilizado em uma autoclave à temperatura de 120 $\pm 1^{\circ} \mathrm{C}$ durante uma hora.

Ao término do ensaio, os frascos foram abertos e os corpos de prova limpos, ao remover o micélio dos fungos, e conduzidos à estufa, mantida nas mesmas condições anteriores até atingir massa constante e, posteriormente, pesados para avaliação da perda de massa.

A avaliação do ensaio foi com base na perda de massa, sendo esta corrigida em função das amostras soterradas em solo esterilizado. Para permitir a comparação com outros trabalhos, foram incluídos no experimento corpos-de-prova de alburno de Pinus sp.

\subsection{Analise dos Resultados}

Com o intuito de detectar as diferenças entre as madeira e as posições na direção medula-casca, os resultados de perda de massa foram analisados estatisticamente. Utilizaram-se as informações da densidade da madeira e do teor de extrativo, para auxiliarem a interpretação dos resultados.

Para comparar a resistência das madeiras estudadas, foi empregado o delineamento inteiramente casualizado, com arranjo fatorial, em que foram analisados os seguintes fatores: madeira, com nove níveis; posição na direção medula-casca, com quatro níveis; e a interação entre os fatores.

Para possibilitar a análise estatística, os dados foram transformados em arco-seno [raiz (perda de massa/ 100)]. Essa transformação dos dados sugerida por Steel e Torrie (1980) foi necessária para a homogeneidade das variâncias. Na análise e avaliação dos ensaios foi empregado o teste de Tukey a 5\% de probabilidade, dos fatores e interação detectados como significativos pelo teste F.

\section{RESULTADOS E DISCUSSÃO}

No Quadro 2, encontram-se os valores médios da densidade das madeiras $\left(\mathrm{g} / \mathrm{cm}^{3}\right)$, do teor de extrativos solúveis em água quente e da perda de massa (\%) sofrida pelos corpos-de-prova submetidos ao ensaio com fungos de podridão-mole.
Observou-se que não há uma boa relação entre o teor de substâncias extraídas em água-quente e a resistência das madeiras ensaiadas. Isso indica que a resistência das madeiras pode estar relacionada a outras classes de extrativos, os quais não foram extraídos em água quente. Resultados semelhantes ocorreram em relação à densidade, em que espécies produtoras de madeiras mais densas nem sempre apresentaram maior resistência à deterioração. Observação semelhante foram feitas por Paes et al. (2001) ao estudarem a resistência de tais madeiras ao cupim xilófago Nasutitermes corniger, em ensaio de preferência alimentar.

O valor da perda de massa para o Pinus sp foi de $24,32 \%$ e, para as madeiras testadas, variou entre as espécies e a posição na direção medula-casca. Tendo as madeiras mais resistentes (aroeira, braúna e pereiro) apresentado perdas de massa de 2,48; 2,98; e 2,71\%, respectivamente. Enquanto que as mais deterioradas (craibeira e cumaru) com perdas de 24,26 e 22,78\%, respectivamente.

A análise de variância dos valores de perda de massa das madeiras estudadas revelou que os fatores espécie, posição e a interação entre eles foram significativos pelo teste $\mathrm{F}$ a $5 \%$ de probabilidade. A interação foi desdobrada e analisada pelo teste de Tukey ( $\mathrm{p} \leq 0,05)$, cujos resultados são apresentados no Quadro 3.

A análise do efeito das espécies em cada posição estudada (Quadro 3) revelou-se, para a posição 1 na madeira, que não houve diferença significativa entre algaroba, angico, craibeira e cumaru. Sendo estas as espécies mais deterioradas pelos fungos de podridãomole existentes no solo utilizado. As menos deterioradas foram aroeira e braúna, que não diferiram da cássia, pau-d'arco, pereiro e angico.

Para a posição 2, notou-se que as madeiras mais deterioradas foram a craibeira e cumaru. As menos atacadas foram o pereiro, pau-d' arco, braúna, aroeira, angico e algaroba, tendo a cássia apresentado resistência intermediária entre as espécies listadas. O cumaru foi semelhante à cássia, angico e algaroba.

Observa-se, na posição 3, que as espécies menos deterioradas foram pereiro, pau-d' arco, aroeira, braúna e algaroba, e as mais atacadas, craibeira, cumaru, cássia e angico. 
Quadro 2 - Valores médios da densidade $\left(\mathrm{g} / \mathrm{cm}^{3}\right)$, do teor de extrativos $(\%)$ e da perda de massa $(\%)$ das madeiras ensaiadas Table 2 - Mean values of wood density $\left(\mathrm{g} / \mathrm{cm}^{3}\right)$, extractive content (\%) and mass loss (\%) of the assayed woods

\begin{tabular}{|c|c|c|c|c|}
\hline $\begin{array}{l}\text { Espécies } \\
\text { Estudadas }\end{array}$ & $\begin{array}{c}\text { Posições no } \\
\text { Tronco }\end{array}$ & $\begin{array}{l}\text { Densidade } \\
\left(\mathrm{g} / \mathrm{cm}^{3}\right)\end{array}$ & $\begin{array}{c}\text { Extrativo em } \\
\mathrm{H}_{2} \mathrm{O} \text { Quente }\end{array}$ & $\begin{array}{c}\text { Perda de } \\
\text { Massa (\%) }\end{array}$ \\
\hline \multirow{4}{*}{1 - Algaroba } & 1-Interna & 0.83 & 5.67 & 18,22 \\
\hline & 2-Med-Int & 0.87 & 4.79 & 8,64 \\
\hline & 3-Med-Ext & 0.88 & 4.93 & 7,22 \\
\hline & 4-Externa & 0.95 & 5.63 & 9,09 \\
\hline \multirow{4}{*}{2 - Angico } & 1-Interna & 0.79 & 7.23 & 8,90 \\
\hline & 2-Med-Int & 0.78 & 7.28 & 8,40 \\
\hline & 3-Med-Ext & 0.80 & 9.60 & 12,84 \\
\hline & 4-Externa & 0.81 & 7.09 & 8,30 \\
\hline \multirow{4}{*}{3 - Aroeira } & 1-Interna & 1.11 & 17.61 & 2,48 \\
\hline & 2-Med-Int & 1.09 & 17.23 & 7,38 \\
\hline & 3-Med-Ext & 1.09 & 18.73 & 5,05 \\
\hline & 4-Externa & 0.94 & 8.05 & 16,9 \\
\hline \multirow{4}{*}{4 - Braúna } & 1-Interna & 1.16 & 16.51 & 2,98 \\
\hline & 2-Med-Int & 1.11 & 22.66 & 3,78 \\
\hline & 3-Med-Ext & 1.11 & 21.41 & 6,32 \\
\hline & 4-Externa & 1.09 & 3.79 & 16,91 \\
\hline \multirow{4}{*}{5 - Cássia } & 1-Interna & 0.99 & 12.73 & 5,80 \\
\hline & 2-Med-Int & 1.02 & 11.24 & 11,81 \\
\hline & 3-Med-Ext & 1.071 & 1.24 & 16,34 \\
\hline & 4-Externa & 0.77 & 7.61 & 12,26 \\
\hline \multirow{4}{*}{6 - Craibeira } & 1-Interna & 0.66 & 3.83 & 18,28 \\
\hline & 2-Med-Int & 0.63 & 3.38 & 24,26 \\
\hline & 3-Med-Ext & 0.68 & 3.51 & 18,72 \\
\hline & 4-Externa & 0.68 & 4.91 & 15,64 \\
\hline \multirow{4}{*}{7 - Cumaru } & 1-Interna & 0.58 & 11.94 & 11,87 \\
\hline & 2-Med-Int & 0.56 & 25.39 & 15,37 \\
\hline & 3-Med-Ext & 0.53 & 15.14 & 17,29 \\
\hline & 4-Externa & 0.49 & 19.60 & 22,78 \\
\hline \multirow{4}{*}{8 - Pau D'arco } & 1-Interna & 1.01 & 7.91 & 4,42 \\
\hline & 2-Med-Int & 0.96 & 7.71 & 5,51 \\
\hline & 3-Med-Ext & 0.95 & 7.79 & 4,97 \\
\hline & 4-Externa & 0.90 & 7.58 & 9,43 \\
\hline \multirow{4}{*}{9 - Pereiro } & 1-Interna & 0.80 & 3.21 & 5,53 \\
\hline & 2-Med-Int & 0.79 & 3.66 & 2,71 \\
\hline & 3-Med-Ext & 0.76 & 4.93 & 3,25 \\
\hline & 4-Externa & 0.80 & 4.71 & 4,38 \\
\hline
\end{tabular}

Quadro 3 - Comparações múltiplas entre médias da perda de massa (\%) causadas pelos fungos nas madeiras estudadas Table 3 - Multiple comparisons among means for loss of woody mass (\%) caused by fungi

\begin{tabular}{lcccc}
\hline Espécies Estudadas & \multicolumn{4}{c}{ Posições na Madeira } \\
\cline { 2 - 5 } & 1 - Interna & 2 - Med. Int. & 3 - Med. Ext. & 4 - Externa \\
\hline 1 - Algaroba & $12,42 \mathrm{Aab}$ & $8,64 \mathrm{Abcd}$ & $7,22 \mathrm{Abcd}$ & $9,09 \mathrm{Abc}$ \\
2 - Angico & $8,90 \mathrm{Aabc}$ & $8,40 \mathrm{Abcd}$ & $12,84 \mathrm{Aabc}$ & $8,30 \mathrm{Abc}$ \\
3 - Aroeira & $2,48 \mathrm{Bc}$ & $7,38 \mathrm{Bcd}$ & $5,05 \mathrm{Bcd}$ & $16,94 \mathrm{Aab}$ \\
4 - Braúna & $2,98 \mathrm{Bc}$ & $3,78 \mathrm{Bcd}$ & $6,32 \mathrm{Bcd}$ & $16,91 \mathrm{Aab}$ \\
5 - Cássia & $5,80 \mathrm{Bbc}$ & $11,81 \mathrm{ABbc}$ & $16,34 \mathrm{Aab}$ & $12,26 \mathrm{ABabc}$ \\
6 - Craibeira & $18,28 \mathrm{Aa}$ & $24,26 \mathrm{Aa}$ & $18,72 \mathrm{Aa}$ & $15,64 \mathrm{Aab}$ \\
7 - Cumaru & $11,87 \mathrm{Aab}$ & $15,37 \mathrm{Aab}$ & $17,29 \mathrm{Aab}$ & $22,78 \mathrm{Aa}$ \\
8 - Pau d'arco & $4,42 \mathrm{Abc}$ & $5,51 \mathrm{Acd}$ & $4,97 \mathrm{Acd}$ & $9,43 \mathrm{Aabc}$ \\
9 - Pereiro & $5,53 \mathrm{Abc}$ & $2,71 \mathrm{Ad}$ & $3,25 \mathrm{Ad}$ & $4,38 \mathrm{Ac}$ \\
\hline
\end{tabular}

As médias seguidas na horizontal da uma mesma letra maiúscula ou na vertical por uma mesma letra minúscula não diferem estatisticamente (Tukey $\mathrm{p} \geq 0,05$ ). 
Foi observado na posição 4 que cumaru, aroeira, braúna e craibeira foram as espécies mais atacadas pelos organismos xilófagos, enquanto pereiro, angico e algaroba foram as menos deterioradas. As espécies cássia e pau-d' arco apresentaram resistência intermediária entre as madeiras citadas.

A craibeira e cumaru foram as mais deterioradas, e o pereiro e pau-d'arco, menos atacadas para todas as posições analisadas. Este resultado está de acordo com Mainieri e Chimelo (1989), que afirmaram serem as madeira de craibeira e cumaru de baixa resistência, e a de pau-d' arco resistente ao apodrecimento. Esses autores também citaram como resistentes, as madeiras de angico e aroeira. Paes et al. (2001) constataram que as madeiras de pereiro e pau-d'arco, apresentaram alta resistência ao cupim xilófago Nasutitermes corniger, em ensaio de preferência alimentar.

Assim, como os fungos causadores da podridãomole atacam madeiras utilizadas em locais expostos a alta umidade, as madeiras de aroeira e braúna (cerne), pereiro e pau-d' arco apresentariam melhor desempenho nessas condições, em comparação com as demais espécies estudadas.

Ao analisar o efeito das posições na peça, observase que algaroba, angico, craibeira, cumaru, pau-d'arco e pereiro não apresentaram diferenças significativas entre as posições analisadas. No entanto, aroeira, braúna e cássia apresentaram diferença significativa dentre as posições, tendo o cerne da aroeira e da braúna (posições 1, 2 e 3) diferido do alburno (posição 4). Já a cássia apresentou a madeira da posição 1 menos atacada que a da posição 3 e a das posições 2 e 4 intermediarias entre as duas citadas.

\section{CONCLUSÕES}

A resistência ao ataque de fungos causadores da podridão-mole não esteve relacionada com a densidade das madeiras estudadas.

A quantidade de substâncias extraídas em águaquente não apresentou boa relação com a resistência das madeiras aos fungos. Isso implica o fato de a resistência das madeiras poder estar relacionada a outras classes de extrativos, que não foram extraídos em água quente.

A craibeira e cumaru foram as madeiras mais atacadas, enquanto pereiro e pau-d' arco, as menos deterioradas em todas as posições analisadas.

R. Árvore, Viçosa-MG, v.29, n.3, p.365-371, 2005
A resistência oferecida pelas madeiras de aroeira e braúna (cerne), pereiro e pau-d'arco aos fungos causadores da podridão-mole que atacam madeiras utilizadas em locais expostos à alta umidade indicou que essas madeiras apresentariam melhor desempenho nessas condições que as demais espécies estudadas.

A resistência oferecida pelas madeiras de algaroba, angico, craibeira, cumaru, pau-d' arco e pereiro, ao contrário do ocorrido com aroeira, braúna e cássia, não foi afetada pela direção medula-casca.

\section{REFERÊNCIAS BIBLIOGRÁFICAS}

\section{AMERICAN SOCIETY FOR TESTING AND}

MATERIALS - (ASTM D - 1413). Standard test

method for wood preservatives by laboratory soilblock cultures. Annual Book of ASTM Standards, v.0410, p. 219-224, 1994.

BEHR, A.E. Decay test methods. In: NICHOLAS, D.D. (Ed.). Wood deterioration and its prevention by preservative treatments: degradation and protection of wood. Syracuse: Syracuse University Press, 1973. v. 1, p.217-246.

CARBALLEIRA LOPEZ, G. A.; MILANO, S. Avaliação da durabilidade natural da madeira e de produtos usados na sua proteção. In: LEPAGE, E. S. (Coord.). Manual de preservação de madeiras. São Paulo: IPT, 1986. v.2, p.473-521.

CAVALCANTE, M. S. Deterioração biológica e preservação de madeiras. São Paulo: IPT, 1982. 40p. (Pesquisa e Desenvolvimento, 8).

GERSONDE, M.; KERNER, W. Soft rot test with soils of different origins Stockholm: The International Research Group on Wood Preservation, 1984. 8p. (Doc. IRG/WP/2226).

HEDLEY, M.E.; BUTCHER, J.A. Protocol for evaluating and approving new wood preservatives. Stockholm: The International Research Group on Wood Preservation, 1985. 7p. (Doc. IRG/WP/2159).

HEDLEY, M.E.; PRESTON, A.F.; CROSS, D.J. Screening of selected agricultural and industrial chemicals as wood preservatives.

International Biodeterioration

Bulletin, v.19, n.1, p.9-18, 1979.

\section{R. Árvore, Viçosa-MG, v.29, n.3, p.365-371, 2005}


HUNT, G. M.; GARRATT, G.A. Wood preservation. 3 ed. New York: McGraw Hill, 1967. 433p.

\section{INSTITUTO DE PESQUISAS TECNOLÓGICAS/ DIMAD - (IPT/DIMAD D - 5). Ensaio acelerado de laboratório para a determinação da eficiência de preservativos contra fungos da podridão-mole. São Paulo: IPT/DIMAD, 1980. 2p. (Publicação IPT, 1157).}

JANKOWSKY, I.P. Potencialidade do creosoto de Eucalyptus spp., como preservativo para madeiras. 1986. 159p. Tese (Doutorado em Engenharia) - Universidade de São Paulo, Escola Politécnica, São Paulo, 1986.

LEPAGE, E.S. Preservativos e sistemas preservativos. In: LEPAGE, E.S. (Coord.) Manual de preservação de madeiras. São Paulo: IPT, 1986, v.1, p.279-342.

MAINIERI, C.; CHIMELO, J.P. Fichas de características das madeiras brasileiras. São Paulo: IPT, 1989. 418p.

McKAIG, P.A. Developments in unsterile soil soft rot testing. Stockholm: The International Research Group on Wood Preservation, 1984. 12p. (Doc. IRG/WP/2210).
PAES, J.B.; MORAIS, V.M.; LIMA,CR. Resistência natural de nove espécies de madeiras do semiárido brasileiro a cupins subterrâneos em ensaio de preferência alimentar. Brasil Florestal, v.20, n. 72, p.59-69, 2001.

PNUD/FAO/IBAMA/UFPB/GOV. PARAIIBA. Diagnóstico do setor florestal do Estado da Paraíba. João Pessoa: PNUD/ FAO/ BRA. 87-007, 1994. 84p.

SAVORY, J.G. Collaborative soft rot tests amended test method. Stockholm: The International Research Group on Wood Preservation, 1972. 7p. (Doc. IRG/WP/208).

SAVORY, J.G.; CAREY, J.K. Collaborative soft rot tests: programme and test method. Stockholm: The International Research Group on Wood Preservation, 1973. 9p. (Doc. IRG/WP/229).

THEDEN, G. The efficacy of wood preservatives towards soft rot determinated by a soil burial procedure. Holz als Roh und Werkstoff, v.19, n.9, p.352-357, 1961.

THORNTON, J.D.; ROBINSON, P.J.; FRENCH, J.R.J. Toxicity of aliphatic acids 1 , against soft rot organisms and Gloeophyllum trabeum.

International Biodeterioration Bulletin, v.13, n.4, p.108-11, 1977.

WILKINSON, J.G. Industrial timber preservation. London: The Rentokil library/ Associated Business-Press, 1979. 532p. 